
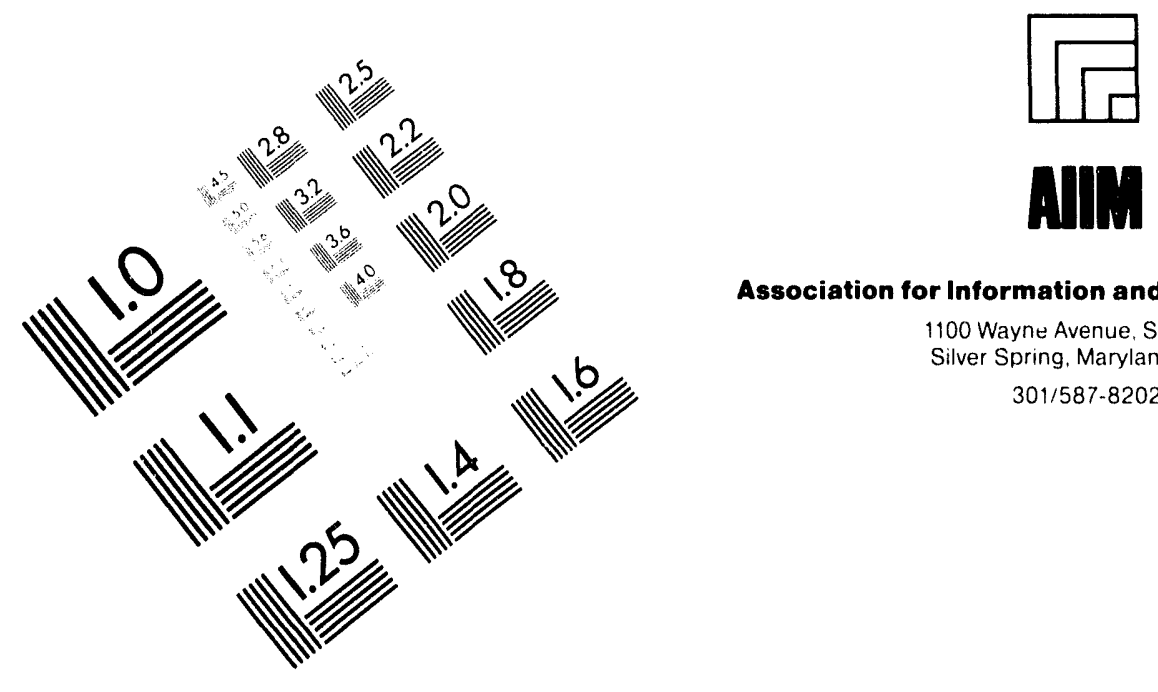

Association for Information and Image Management

1100 Wayne Avenue, Suite 1100

Silver Spring. Maryland 20910

301/587-8202

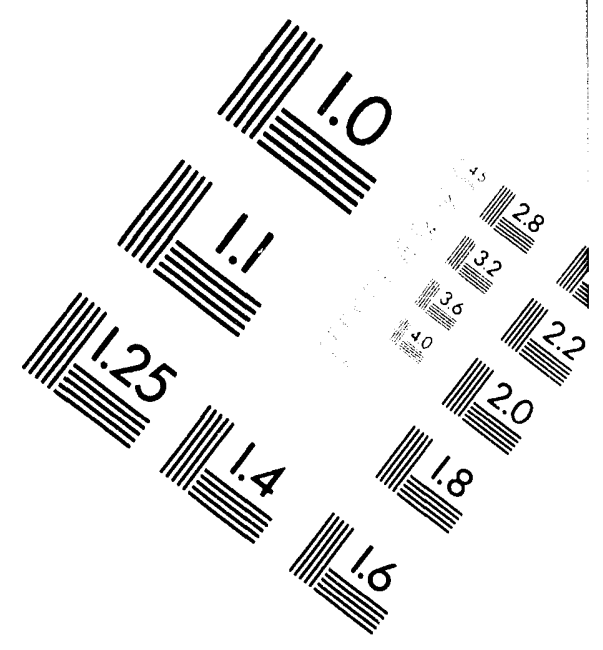

\title{
Centimeter
}

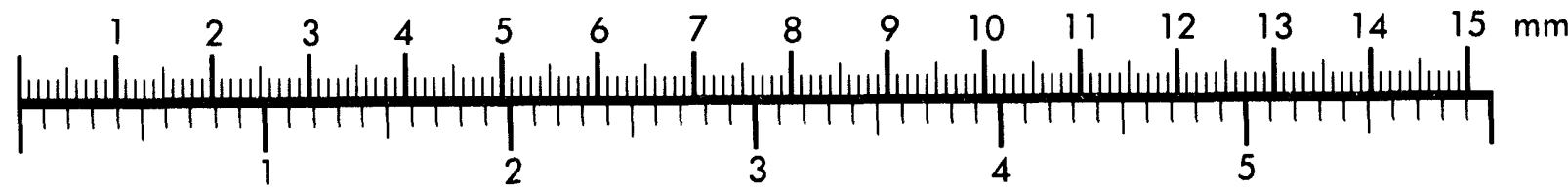

Inches
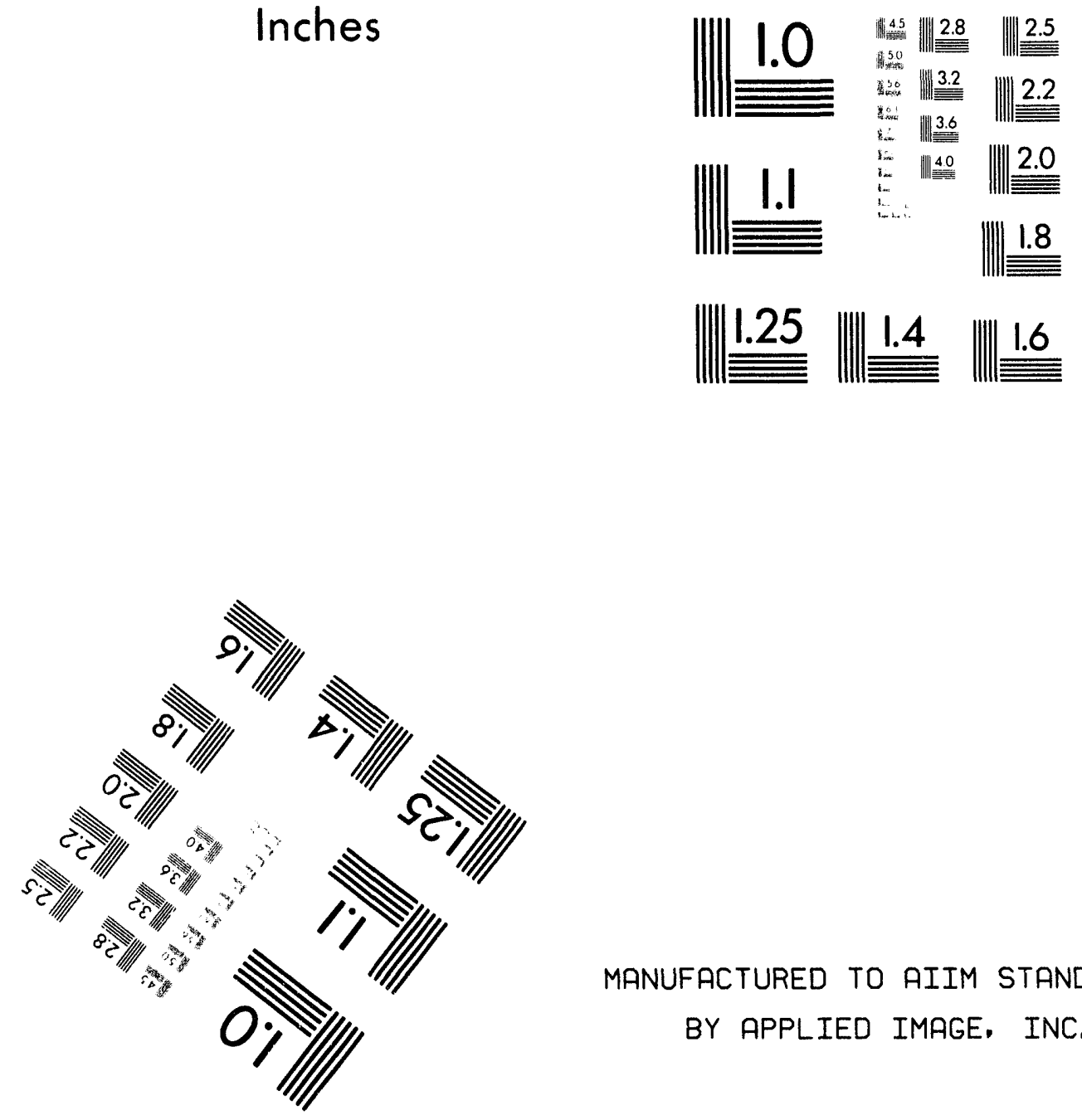

MANUFACTURED TO AIIM STANDARDS

BY APPLIED IMAGE, INC.

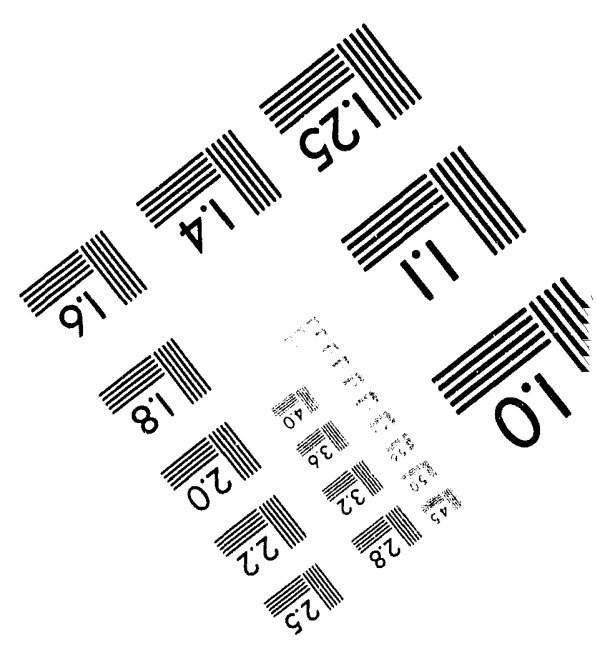



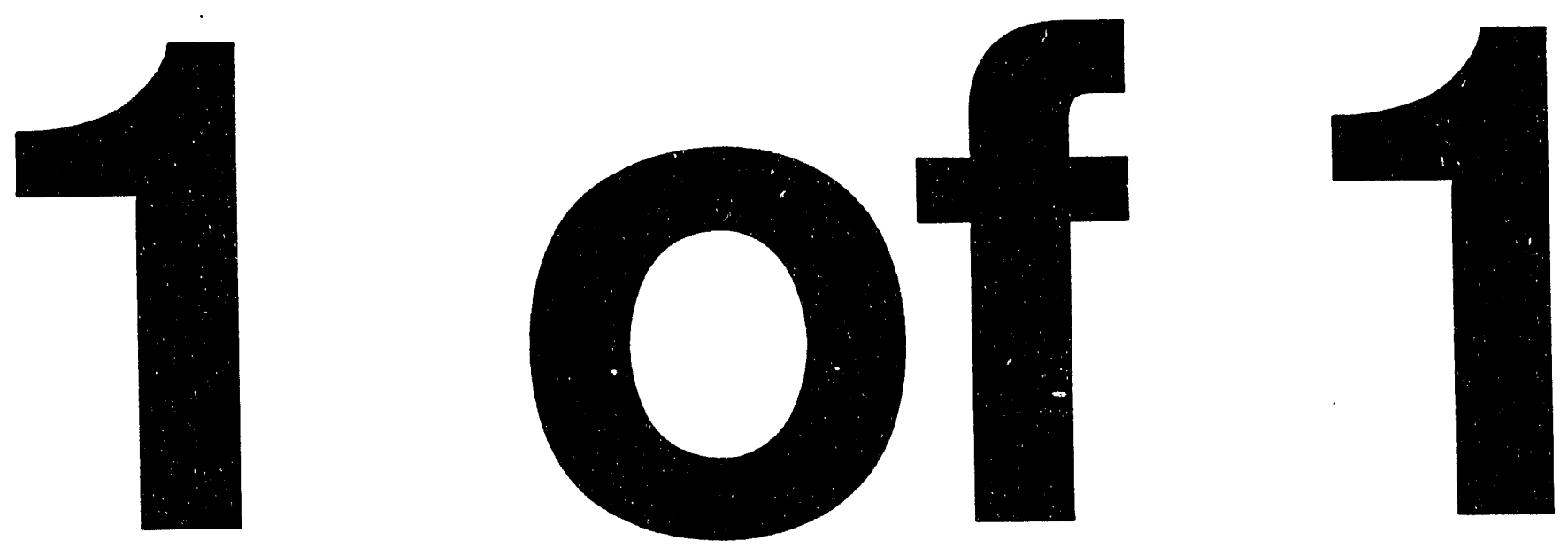


\title{
CONF-930999--11
}

Talk presented at NATO Advanced Study Institute on "Hot and Dense Nuclear Matter," Bodrum, Turkey (1993). Sept. 26-0ct.9, i993

\section{BOLTZMANN-LANGEVIN TRANSPORT MODEL FOR HEAVY-ION COLLISIONS}

\author{
Sakir Ayik \\ Tennessee Technological University, Cookeville, TN 38505, USA \\ and \\ Joint Institute for Heavy-Ion Research, Oak Ridge, TN 37831, USA
}

\begin{abstract}
Heavy-ion collisions at intermediate energies exhibit catastrophic phenomena which requires descriptions based on stochastic transport models. First, the BoltzmannLangevin model, which provides an example of such stochastic approaches, is briefly described. Then, a projection method for obtaining numerical solutoins of the Boltzmann-Langevin equation is discussed. Finally, some applications of the model to heavy-ion collisions are presented.
\end{abstract}

\section{INTRODUCTION}

Many aspects of heavy-ion collisions can be described by means of the one-body transport models. In these transport models, one deals with a reduced description in terms of the single-particle density, rather than the full many-body information. These rodels in semi-classical limit with a Boltzmann-Uehling-Uhlenbeck (BUU) form of a collision term has been very successful in describing a large variety of observables associated with heavy-ion collisions at intermediate energies [1-2]. The average description provided by the BUU model is well suited for processes involving small density fluctuations. However, for processes involving large density fuctuations, for example near instabilities and bifurcations, such an average description is inadequate. In these situations the stochastic transport models may provide a more appropriate basis for describing the dynamical evolution. In these stochastic approaches, the one-body transport models are improved beyond the mean-field approximation by incorporating the high order correlations in a statistical approximation, analogous to the treatment of the Brownian motion. The recently developed Boltzmann-Langevin (BL) model constitutes an example of such stochastic transport approaches [3-5], and it is therefore a promising model for describing catastrophic phenomena, such as phase transitions and nuclear multifragmentations.

In section 2, we briefly describe the BL model. In section 3, we discuss a moment expansion of the BL equation, which is useful for small fluctuations around the average trajectory. In section 4, we describe a projection method for obtaining approximate numerical solutions of the BL equation. In section 5 , we present some applications to heavy-ion collisions in which we investigate the dynamics of density fluctuations, and the influence of momentum space fluctuations of kaon production cross-sections at subthreshold energies. Finally, in section 6 , we make some remarks on the further developments of the model and give some conclusions.

\section{BOLTZMANN-LANGEVIN EQUATION}

The BUU model determines the evolution of the average phase-space density $f(r, p, t)$ in the semi-classical limit according to the transport equation,

$\left(\frac{\partial}{\partial t}+\nabla \cdot \nabla_{r}-\nabla_{r} U \cdot \nabla_{p}\right) f(r, p, t)=K(f)$.

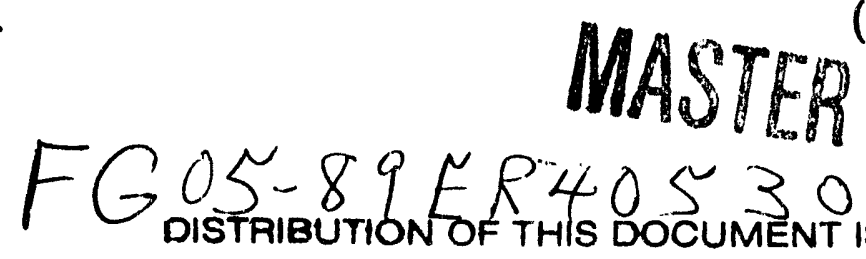




\section{DISCLAIMER}

This report was prepared as an account of work sponsored by an agency of the United States Government. Neither the United States Government nor any agency thereof, nor any of their employees, makes any warranty, express or implied, or assumes any legal liability or responsibility for the accuracy, completeness, or usefulness of any information, apparatus, product, or process disclosed, or represents that its use would not infringe privately owned rights. Reference herein to any specific commercial product, process, or service by trade name, trademark, manufacturer, or otherwise does not necessarily constitute or imply its endorsement, recommendation, or favoring by the United States Government or any agency thereof. The views and opinions of authors expressed herein do not necessarily state or reflect those of the United States Government or any agency thereof. 
Here the left-hand-side describes the Vlasov propagation determined in terms of the nuclear mean-field $U(f)$. On the right-hand-side, $K(f)$ denotes a binary collision term given by

$$
\begin{aligned}
K(f)=\int d p_{2} d p_{3} d p_{4} W(12 ; 34)[(1-f) & \left(1-f_{2}\right) f_{3} f_{4} \\
& \left.-f f_{2}\left(1-f_{3}\right)\left(1-f_{4}\right)\right]
\end{aligned}
$$

where $f_{j} \equiv f\left(r, p_{j}, t\right)$ and $W(12 ; 34)$ denotes the transition rate between two-particle states, which can be expressed in terms of the in-medium nucleon-nucleon scattering crosssection [1-2].

In energetic heavy-ion collisions, the nuclear system decays into a great variety of final states. In contrast to the experimental situations, the BUU model yields a unique (deterministic) trajectory for a given initial condition, which may be considered as an average over all possible final states (ensemble averaging), and the dynamical branching is not allowed. In particular the BUU description becomes worse when the spreading of the trajectories of the single-particle densities associated with the final states is large. This severe limitation follows from the independent collision approximation employed in the derivation of the BUU model. The derivation of the BUU model involves two levels of approximations. The first one is the truncation of dynamics at a two-body level, which is a good approximation for a sufficiently dilute system. However, this is not sufficient. A more drastic approximation must be introduced by neglecting the correlations between subsequent binary collisions, which is usually referred to as the molecular chaos assumption. As a result, the fluctuations are not propagated and the independent binary collisions always drive the system along the average trajectory without breaking the initial symmetries of the system.

In order to describe the fluctuations, i.e., the spreading of trajectories of the single-particle densities, the effects of correlations must be restored into the equation of motion in some ways. The residual interactions, in general, play two different roles: (i) producing dissipation by randomizing the momentum distribution via binary collisions and (ii) inducing fluctuations by propagating correlations in the phase-space. By incorporating these two effects of the residual interactions into the equation of motion, one obtains stochastic transport equations, which can be studied in various representations [3-5]. In the semi-classical limit the equation of motion for the fluctuating phase-space density $\hat{f}(r, p, t)$ takes the form,

$$
\left[\frac{\partial}{\partial t}+\mathbf{v} \cdot \nabla_{r}-\nabla_{r} \hat{U} \cdot \nabla_{p}\right] \hat{f}(r, p, t)=K(\hat{f})+\delta K(r, p, t) .
$$

which is referred to as the BLE. Here, the collision term $K(\hat{f})$ has the usual BUU form but is expressed in terms of the fluctuating density $\hat{f}(r, p, t)$. On the right-hand-side, in addition to the usual collision term, it appears an additional term $\delta K(r, p, t)$, which is called the fluctuating collision term. The fluctuating collision term arises from correlations not accounted for by the collision term. As a matter of fact, such an additional term always arises in transport theory whenever we deal with a reduced description and it describes the coupling to the degrees of freedom, which are not explicitly considered [6-7]. The fluctuating collision term has many properties which are similar to the random force in a typical Langevin equation: (i) It varies rapidly in time with a characteristic time in the order of the duration time of a two-body collision. (ii) It is nearly impossible to calculate the fluctuating collision term explicitly because it is equivalent for exactly solving the many-body problem. (iii) It vanishes on the average with the molecular chaos assumption, hence, does not appear in the average 
description of the BUU model. The BLE contains full information about dissipation and fluctuation properties of the single-particle density. However, in order to have a tractable model, we need to introduce approximations. In analogy with the Brownian motion, it is assumed that eq. (2.3) describes a stochastic process in which the whole density is a stochastic variable and the fluctuating collision term acts like a random force [8]. In such a stochastic description the fluctuating collision term is characterized by a correlation function,

$$
<\delta K(r, p, t) \delta K\left(r^{\prime}, p^{\prime}, t^{\prime}\right)>=C\left(p, p^{\prime}\right) \delta\left(r-r^{\prime}\right) \delta\left(t-t^{\prime}\right)
$$

which is assumed to be local in space and time without the memory effects. With a specified correlation function, the BLE becomes a well-defined stochastic transport equation for the fluctuating single-particle density. It provides a probabilistic description in contrast to the deterministic description of the BUU model. The BLE has many solutions with a given initial condition. Each solution produces an event and many solutions are needed for describing a collision process.

The BLE was first proposed by Bixon and Zwanzig in order to describe the hydrodynamic fluctuations [9]. They evaluate the correlation function $C\left(p, p^{\prime}\right)$ in equilibrium using the fluctuation-dissipation theorem as an input. As a result, their model is valid only for classical systems near equilibrium. In order to describe nonequilibrium fluctuations in quantal systems, Ayik and Gregoire calculate the correlation function directly in non-equilibrium within a weak-coupling approximation [3]. In the semi-classical limit, the correlation function is given by

$$
\begin{aligned}
& C\left(p, p^{\prime}\right)=\int d p_{3} d p_{4} W\left(11^{\prime} ; 34\right)\left[f_{1} f_{1}^{\prime}\left(1-f_{3}\right)\left(1-f_{4}\right)+\left(1-f_{1}\right)\left(1-f_{1}^{\prime}\right) f_{3} f_{4}\right] \\
& -2 \int d p_{2} d p_{4} W\left(12 ; 1^{\prime} 4\right)\left[f_{1} f_{2}\left(1-f_{1}^{\prime}\right)\left(1-f_{4}\right)+\left(1-f_{1}\right)\left(1-f_{2}\right) f_{1}^{\prime} f_{4}\right] \\
& +\delta\left(p-p^{\prime}\right) \int d p_{2} d p_{3} d p_{4} W(12 ; 34)\left[f_{1} f_{2}\left(1-f_{3}\right)\left(1-f_{4}\right)+\left(1-f_{1}\right)\left(1-f_{2}\right) f_{3} f_{4}\right]
\end{aligned}
$$

where $W(12 ; 34)$ is the same transition rate which enters into the collision term and $f_{j}=$ $f\left(r, p_{j}, t\right)$ is the locally averaged single-particle density. The correlation function is entirely determined by the one-body properties and is closely related to the collision term. Aside from the mean-field and the nucleon-nucleon cross-section, no other information is needed for describing the fluctuations. The fluctuation and dissipation properties of density, which are described by the collision term and the correlation function, are not independent properties, but must be related to each other (as in any relaxation process) through a fluctuation-dissipation theorem. Therefore, the close relationship between the correlation function and the collision term can be regarded as a fluctuation-dissipation theorem associated with the stochastic evolution of the singleparticle density. The BLE satisfies the conservation laws of total energy, total momentum, and total particle number. In contrast to the Brownian motion in which the energy conservation is satisfied on the average, each event of the BLE respects the conservation laws. This property follows from the fact that the fluctuating collision term in the BLE is an internal noise and the correlation function satisfies certain sum rules. The correlation function $C\left(p, p^{\prime}\right)$ is valid for large fluctuations in non-equilibrium. For small fluctuations around equilibrium, it reproduces the known result of Bixon and Zwanzig. 


\section{MOMENT EXPANSION}

The BLE generates an ensemble of phase-space densities $\{\hat{f}(r, p, t)\}$ and the proper object of study is therefore the distribution of such densities. Useful information on the deviations $\delta f(r, p, t)$ of the individual densities from the ensemble averaged one $f(r, p, t)=\langle\hat{f}(r, p, t)\rangle$ is expressed by the correlation function,

$\sigma\left(\mathbf{r}, \mathbf{p} ; \mathbf{r}^{\prime}, \mathbf{p}^{\prime} ; \mathbf{t}\right)=\left\langle\delta f(\mathbf{r}, \mathbf{p}, \mathbf{t}) \delta f\left(\mathbf{r}^{\prime}, \mathbf{p}^{\prime}, \mathbf{t}\right)\right\rangle$.

The correlation function $\sigma\left(\mathbf{r}, \mathbf{p} ; \mathbf{r}^{\prime}, \mathbf{p}^{\prime}\right)$ can be used to calculate the covariances between any two one-body observables $A(r, p)$ and $B(r, p)$,

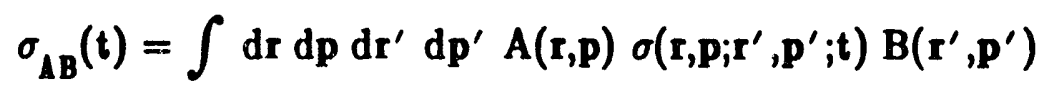

which vanishes if one of the observables is dynamically conserved. By linearizing the BLE around the average trajectory for small fluctuations, it is possible to derive closed equations of motion for the first and second moments. The equation for the first moment, i.e., the average density, is the BUU equation. The equation of motion for the second moment, i.e., the correlation function (3.1), becomes

$$
\begin{aligned}
{\left[\frac{\partial}{\partial t}+\nabla_{1} \cdot \nabla_{1}+\mathbf{v}_{1}^{\prime} \cdot \nabla_{1}^{\prime}\right] \sigma\left(\mathbf{r}, \mathbf{p} ; \mathbf{r}^{\prime}, \mathbf{p}^{\prime}\right) } & =\mathbf{C}\left(\mathbf{r}, \mathbf{p} ; \mathbf{r}^{\prime}, \mathbf{p}^{\prime}\right) \\
& +\mathbf{I}_{1} \cdot \sigma\left(\mathbf{r}, \mathbf{p} ; \mathbf{r}^{\prime}, \mathbf{p}^{\prime}\right)+\mathrm{I}_{1}{ }^{\prime} \cdot \sigma\left(\mathbf{r}, \mathbf{p} ; \mathbf{r}^{\prime}, \mathbf{p}^{\prime}\right)
\end{aligned}
$$

where I represents the linearized collision operator evaluated with the average density. On the left, the terms due to the mean-field are dropped by assuming a constant potential, which can always be added to the equation of motion.

The information contained in eqs. (2.1) and (3.3), namely, the ensemble averaged evolution and the character of the fluctuations around this average, is useful in situations when the bundle of trajectories remains reasonably well confined around the average. This is always true for stable modes. When the instabilities are present, the trajectories branch out to very different configurations at large times that the overall average does not make sense. However, for sufficiently short times, the moment approximation provides a useful tool for addressing the early development of the unstable collective modes [10].

In a particular situation when the system remains close to a local equilibrium, one linearizes the BLE around the local equilibrium, and the linearized equation can be used to extract transport coefficients associated with macroscopic variables [11]. As an example here we consider that a system is initially uniform and in equilibrium at a temperature $T$ with a phase-space density $f_{0}(\epsilon)$. We can calculate the magnitude of small density fluctuations by linearizing the BLE (2.3) around equilibrium, $\hat{f}(r, p, t)=$ $f_{0}(\epsilon)+\delta f(r, p, t)$,

$\frac{\partial}{\partial t} \delta f+\nabla \cdot \nabla_{\mathrm{r}} \delta f+\nabla_{\mathrm{r}} \delta U \cdot \nabla_{\mathrm{p}} \mathrm{f}_{0}=\mathrm{I}_{0} \cdot \delta f+\delta \mathrm{K}$

where $I_{0}$ is the linearized collision operator evaluated with the equilibrium density $f_{0}$. This equation is easily solved for the density fluctuations by expanding $\mathscr{f}(\mathbf{r}, \mathbf{p}, t)$ on plane waves,

$\delta_{p}(\mathbf{k}, \omega)=\int \mathrm{dr} d t \mathrm{e}^{-\mathrm{i}(\mathbf{k} \cdot \mathbf{r}-\omega t)} \delta f(\mathbf{r}, \mathbf{p}, t)$ 
When integrated over momentum $\mathbf{p}$, this relation gives the Fourier coefficients $\delta$ n $(\mathbf{k}, \omega)$ of the local density fluctuations. Then, in weak-damping limit, the density correlation function can be expressed as

$<\delta \mathbf{n}(\mathbf{k}, \omega) \delta n\left(\mathbf{k}^{\prime}, \omega^{\prime}\right)>=(2 \pi)^{4} \delta\left(\mathbf{k}+\mathbf{k}^{\prime}\right) \delta\left(\omega+\omega^{\prime}\right) \sigma(\mathbf{k}, \omega)$

with

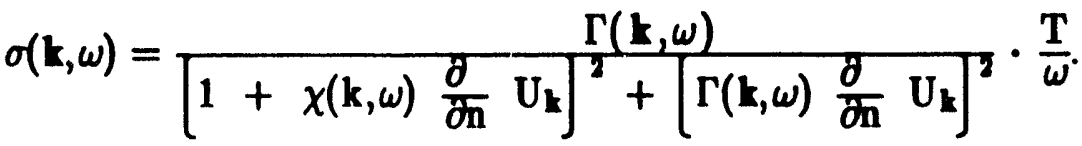

Here $U_{k}$ denotes the Fourier transform of the mean-field, and the quantities $\chi(\mathbf{k}, \omega)$ and $\Gamma(\mathbf{k}, \omega)$ are given by

$\chi(\mathbf{k}, \omega)=\int \frac{\mathrm{dp}}{(2 \pi)^{3}} \frac{\mathbf{k} \cdot \nabla \mathbf{f}_{0}}{\omega-\mathbf{k} \cdot \nabla}$

$\Gamma(\mathbf{k}, \omega)=\frac{\omega}{T} \int d p_{1} d p_{2} d p_{3} d p_{4} W(12 ; 34)\left[\frac{\Delta Q}{2}\right]^{2} f_{1} f_{2}\left(1-f_{3}\right)\left(1-f_{4}\right)$

with $\Delta Q=Q_{1}+Q_{2}-Q_{3}-Q_{1}, Q_{j}=\left(1-\mathbf{k} \cdot \nabla_{j}\right)^{-1}$. The equilibrium density fluctuations in a given mode $\mathbf{k}$ is determined by integrating $\sigma(\mathbf{k}, \omega)$ over frequency,

$\sigma(\mathbf{k})=\int \frac{\mathrm{d} \omega}{2 \pi} \sigma(\mathbf{k}, \omega)=\frac{\mathbf{n}_{0} \mathbf{k}^{2}}{\mathrm{~m} \omega_{\mathbf{k}}} \cdot \frac{\mathbf{T}}{\omega_{\mathbf{k}}}$

where $\mathrm{n}_{0}$ is the equilibrium density and the frequencies $\omega_{k}$ of the collective modes are determined by the dispersion relation, $1+\chi(k, \omega) \partial U_{k} / \partial n=0$. This result is valid in semi-classical limit, $T>w_{k}$. At low temperatures, $T \leq \omega_{k}$, the quantal effects become important and should be incorporated into the BLE in the form of memory effects. After this modification, the factor $\mathrm{T} / \omega_{\mathrm{k}}$ in eq. (3.10) is replaced by $\operatorname{coth}\left(\omega_{k} / 2 \mathrm{~T}\right)[11]$.

\section{PROJECTION METHOD FOR NUMERICAL SIMULATION}

The stochastic character of nuclear dynamics is especially important when instabilities occur, since different possible trajectories may lead towards configurations that differ drastically from one another. For a quantitative treatment of such catastrophic processes, one must carry out numerical simulations of the BLE. Randrup and coworkers have developed a lattice simulation method by treating the two-body transition rates as random variables characterized by a Poisson distribution [12]. However, a direct simulation of this manner is not very practical and propagates too much detailed information, which is not needed for describing gross properties of the density fluctuations. Therefore one has to develop methods for obtaining approximate solutions of the BLE.

In order to describe the gross properties of density fluctuations, it may be sufficient to propagate the fluctuations associated with a few low order multipole moments of the momentum distribution. As can be seen from the fluid dynamical description, the evolution of density is coupled to the fluctuation-dissipation mechanism through the momentum flow tensor, which is nothing but the local quadrupole moment of the momentum distribution. Based on this observation, Ayik and coworkers have proposed a simulation method by projecting fluctuations on the local multipole moments of the momentum distribution [13], 
$\hat{Q}_{\mathbf{L}}(\mathbf{r}, \mathbf{t})=\int d p Q_{L}(\mathbf{p}) \hat{f}(\mathbf{r}, \mathbf{p}, \mathbf{t})$.

Here $Q_{L}(p)$ is the multipole moment operator of order $L$ in the momentum space which is a $2 L+1$ dimensional vector with components $Q_{L M}(p)$. The fluctuations of the multipole moments are characterized by a diffusion matrix, which can be deduced from the microscopic correlation matrix $C\left(p, p^{\prime}\right)$ as

$C_{L L^{\prime}}(\mathbf{r}, t)=\int d p d p^{\prime} Q_{L}(p) Q_{L}\left(p^{\prime}\right) C\left(p, p^{\prime}\right)$

$=\int d p_{1} d p_{2} d p_{3} d p_{4} \Delta Q_{L} \Delta Q_{L^{\prime}}, W(12 ; 34) f_{1} f_{2}\left(1-f_{3}\right)\left(1-f_{4}\right)$

with $\Delta Q_{L}=Q_{L}\left(p_{1}\right)+Q_{L}\left(p_{2}\right)-Q_{L}\left(p_{3}\right)-Q_{L}\left(p_{4}\right)$. This quantity determines the early growth rate of the fluctuations of $Q_{L}$ 's, and it can be easily computed at each time step with the pseudo particle simulation. The idea is now to simulate the evolution of $Q_{L}$ 's by performing a multi-dimensional random walk in accordance with the time and position dependent diffusion matrix given above. Then, a single dynamical trajectory can be determined according to the following algorithm: (i) Starting with a definite density $\hat{f}(r, p, t)$ at time $t$, its average evolution and the elements of the diffusion matrix are calculated during the time step $\Delta t$ with the particle simulation, yielding $f(r, p, t+$ $\Delta t$ ) and $C_{L L^{\prime}}(r, t)$. (ii) In the second step, the fluctuations of the multipole moments are determined according to a multi-dimensional Langevin equation,

$\hat{Q}_{L}(r, t+\Delta t)=Q_{L}(r, t+\Delta t)+\sum_{L^{\prime}}[\sqrt{\Delta t C(r, t)}]_{L^{\prime}}, \hat{W}_{L^{\prime}}$

Here $Q_{\mathbf{L}}(r, t+\Delta t)$ is the multipole moment associated with the locally averaged density $f(r, p, t+\Delta t)$, and the quantity in the second term is the square-root of the diffusion matrix multiplied by the independent Gaussian random numbers $\hat{W}_{L}$ with unit variance and zero mean for each multipole moment. The square-root of the diffusion matrix is defined in a standard way in terms of the orthogonal transformation which diagonalizes $\mathrm{C}_{\mathrm{LL}^{\prime}}(\mathbf{r}, \mathbf{t})[8]$. (iii) Finally, the fluctuations are inserted into the phase-space by

scaling the local momentum distribution to the new values of $\hat{Q}_{L}{ }^{\prime} s, f(r, p, t+\Delta t) \rightarrow$ $\hat{f}(r, p, t+\Delta t)$. This procedure is repeated at each time step. In the oractical applications of this method, the multipole space must be truncated to a reasonable size. We expect that the propagation of fluctuations by the quadrupole scaling abo re should provide a good approximation for the gross properties of density fluctuations.

\section{APPLICATIONS TO HEAVY-ION COLLISIONS}

\subsection{Density Fluctuations}

The BLE approach provides a useful basis for studying dynamics of density fluctuations in nuclear collisions at intermediate energies and for investigating possible connections between the reaction mechanism of the multifragmentation process and the 
nuclear matter equation of state. The multifragmentation process is usually associated with the instabilities in the spinodal region. In the spinodal region the nuclear system becomes unstable with respect to density fluctuations, and that causes the system to break up into clusters. In order to investigate the density fluctuations in heavy-ion collisions, a number of calculations has been carried out [13]. In most of these calculations the BLE events are determined in the lowest order approximation by propagating only the fluctuations associated with the z-component of the quadrupole moment of the momentum distribution, $\mathbf{Q}_{20}(\mathbf{p}) \equiv \mathbf{Q}_{2}(\mathbf{p})=2 \mathrm{p}_{\mathrm{z}}^{2}-\mathbf{p}_{\mathbf{x}}^{2}-\mathbf{p}_{\mathbf{y}}^{2}$. In this case, eq. (4.3) reduces to a one-dimensional Langevin equation involving only a single diffusion coefficient $C_{22}(t) \equiv C_{2}(t)$. In these calculations a simplified three parameters $\left(t_{0}, t_{3}, \gamma\right)$ Skyrme interaction is employed, which gives an incompressibility modulus of $200 \mathrm{MeV}$, and an energy dependent nucleon-nucleon cross-section together with phenomenological medium effects is used [14]. The computations are performed with 20 pseudo particles per physical nucleon and the collision integral is evaluated by means of, so-called, the full ensemble technique. First, we consider the fluctuations associated with the momentum space and calculate the mean value $\left\langle Q_{2}\right\rangle$ and the variance $\sigma_{2}^{2}=$ $\left\langle Q_{2}^{2}\right\rangle-\left\langle Q_{2}\right\rangle^{2}$ of the total quadrupole moment, which provide measures for the energy dissipation and the fluctuations in the momentum space, respectively. As an example, Figure 1 shows the time evolution of the mean value $\left\langle Q_{2}\right\rangle$, the diffusion coefficient $\mathrm{C}_{2}(t)$, the variance $\sigma_{2}(t)$ of the total quadrupole moment of the momentum distribution and the collision rate in ${ }^{12} \mathrm{C}+{ }^{12} \mathrm{C}$ collisions at various energies. The mean value of the quadrupole moment exhibits a typical relaxation pattern. But the effects of the binary collisions do not show up in $\mathbf{Q}_{2}$ immediately after touching, which occurs at about 5-10 $\mathrm{fm} / \mathrm{c}$ in the figure. Due to the effects of the mean-field, there is a time delay of about 15-20 $\mathrm{fm} / \mathrm{c}$ during which the mean value of the quadrupole moment does not show up any damping, but in fact may increase as a result of the initial compression and the diabatic shift of the single-particle energies. On the other hand, the dispersion $\sigma_{2}$ associated with the quadrupole moment starts growing immediately after touching and reaches large values before the mean value of the quadrupole moment exhibits any sizeable damping. The diffusion coefficient $C_{2}$ is concentrated during the early stages of the collision and its peak value is much larger than the asymptotic background. As a result, the dispersion $\sigma_{2}$ exhibits a bump during the early stages of the collision, which is a characteristic behavior of a strong transient effect. There are strong correlations between the dissipation rate, the collision rate and the magnitude of fluctuations. The dissipation rate and the magnitude of fluctuations are large when the collision rate is high. We also notice that the magnitude of fluctuations increases for increasing energy. This follows from the fact that with increasing bombarding energy, the Pauli blocking becomes less effective and, consequently, the available phase-space for decay becomes larger, which increases the dissipation rate and the magnitude of fluctuations. Also, calculations are carried out in which the BL events are determined by the quadrupole plus octupole scaling. The results of these calculations for the quadrupole moment are similar to those obtained by the quadrupole scaling alone. However, the situation is different for the octupole moment. The scaling of the quadrupole moment alone does not generate fluctuations in the octupole mode, and as a result its variance remains small. In the case of the quadrupole plus octupole scaling, the fluctuations in the octupole mode is also propagated and its variance exhibits the characteristic bump. These calculations indicate that large dynamical fluctuations are introduced into the momentum space during early non-equilibrium stages of the collision and the amplitude of fluctuations increases with increasing energy.

The fluctuations in tie momentum space are subsequently propagated by the mean-field into density. If the system enters into the spinodal region, the fluctuations trigger the instabilities causing the system to break-up into clusters. In a recent work [15], the BL model has been applied to investigate the multifragmentation processes in 

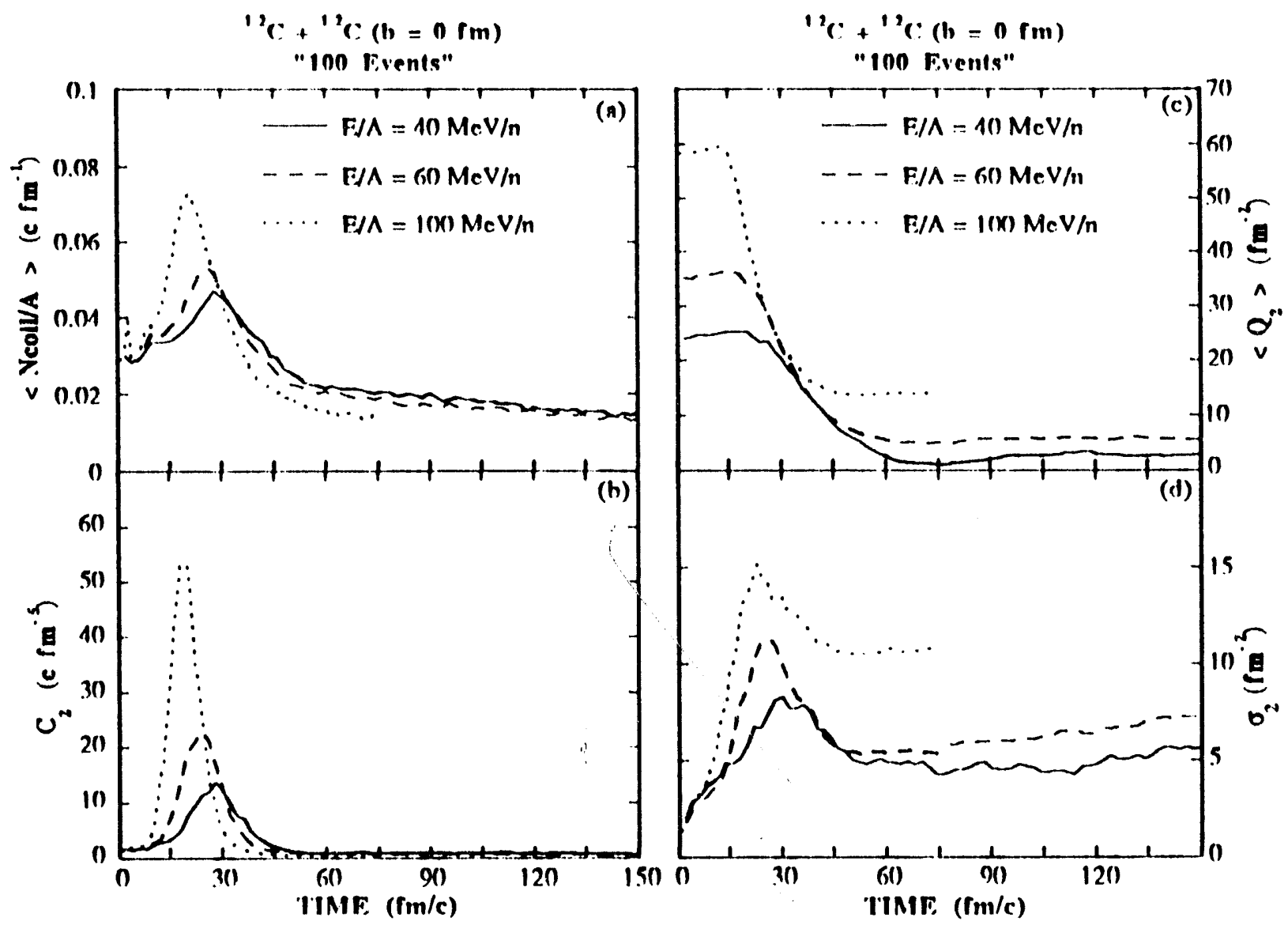

Figure 1. Time evolution of the collision rate (a), the diffusion coefficient (b), the mean value (c) and the variance (d) associated with the total quadrupole moment of the momentum distribution in ${ }^{12} \mathrm{C}+{ }^{12} \mathrm{C}$ collisions at bombarding energies of 40 (solid lines), 60 (dashed lines) and 100 (dotted lines) MeV per nucleon. Taken from ref. [11].

${ }^{10} \mathrm{Ca}+{ }^{10} \mathrm{Ca}$ collisions at bombarding energies of 60 and $90 \mathrm{MeV} / \mathrm{n}$. In these calculations the final state of each event is analyzed using a coalescence model, and the average properties of the intermediate mass fragments are deduced. These calculations indicate that the gross properties of the intermediate mass fragments produced in ${ }^{40} \mathrm{Ca}$ $+{ }^{10} \mathrm{Ca}$ collisions are qualitatively similar to those observed in heavier systems by the MSU group [16] and by the ALADIN collaboration [17].

\section{2. $\quad K+$ Production at Sub-Threshold Energies}

On the basis of the BL approach one can study the rare processes occurring in heavy-ion collisions and investigate the effects of correlations on the sub-threshold particle production and the hard photon emission. The strong transient behavior of the momentum space described in the previous section could make an important effect on the particle production mechanism in heavy-ion collisions below threshold energies. When the fluctuations are large, i.e., during the time interval where the dispersion of the quadrupole moment is going through a maximum, a fraction of the BL events may have sufficient energy to produce mesons below threshold energies, which is otherwise not 
possible in the average description of the BUU model. In addition, the multiparticle interactions involving more than two nucleons provide an efficient mechanism to produce sub-threshold particles [18]. Both effects should be incorporated into the crosssection calculations. Here, we consider the effects of fluctuations and discuss the calculations carried out for the subthreshold $\mathrm{K}^{+}$production cross-section in the BL approach and compare the results with those obtained in the average description of the BUU model [19].

At energies below $1.0 \mathrm{GeV} / \mathrm{n}$, kaons are produced mainly in the elementary baryon-baryon collisions and the invariant $\mathrm{K}^{+}$production cross-section is evaluated by folding the elementary cross-section with the momentum distribution of the colliding baryons [20],

$E \frac{d^{3}}{d p^{3}} \sigma_{\mathbf{r}^{+}}=\Sigma \int_{c} 2 \pi b d b \int d t \int d r d p d p^{\prime}\left|r-\nabla^{\prime}\right| E \frac{d^{3}}{d p^{3}} \sigma_{c} \cdot F$

Here $\mathrm{d}^{3} \sigma_{c} / \mathrm{dp}^{3}$ denotes the elementary cross-section in channels "c", $\mathrm{B}+\mathrm{B} \rightarrow \mathrm{B}+\mathrm{K}^{+}+\mathrm{Y}$, with $B$ as either a nucleon or a delta and $Y$ representing a $\Lambda$ - or a $E$-hyperon. The pion channels are also included in the calculations. In the BUU approach the factor F is given in terms of the ensemble averaged single-particle density by

$F \rightarrow F_{\text {BUU }}=f(r, p, t) f\left(r, p^{\prime}, t\right)\left[1-f\left(r, p^{\prime \prime}, t\right)\right]$.

In the BL approach, the cross-section is evaluated in the same way, but the factor $\mathrm{F}$ is determined as an average over an ensemble of BL events,

$F \rightarrow F_{B L E}=\left\langle\hat{f}(r, p, t) \grave{f}\left(r, \mathbf{p}^{\prime}, t\right)\left[1-\hat{f}\left(r, \mathbf{p}^{\prime \prime}, t\right)\right]\right\rangle$.

An ensemble of BL events is determined using the projection method in the lowest order approximation with quadrupole scaling as discussed in Section 4, and the $\mathrm{K}^{+}$production cross-section in both the BUU and the BL approaches are calculated with the input data of ref. [21]. In order to improve the statistics of the numerical simulations at low bombarding energies, also a model calculation is performed by parameterizing the fluctuating momentum distribution in terms of the z-component of the total quadrupole moment $Q$ of the momentum distribuion $\hat{f}(r, p, t) \rightarrow f_{Q}(r, p, t)$, assuming a Gaussian form for the distribution function $P(Q)$ of the quadrupole moments. In this case, the factor $F$ in eq. (5.1) becomes

$F \rightarrow F_{G}=\int d Q P(Q) f_{Q}(r, p, t) f_{Q}\left(r, p^{\prime}, t\right)\left[1-f_{Q}\left(r, p^{\prime \prime}, t\right)\right]$.

In this Gaussian model, the mean value $\langle Q\rangle$ and the variance $\sigma_{Q}$ of the distribution function $P(Q)$ as well as the momentum distribution $f_{Q}(r, p, t)$ for each $Q$-bin, are extracted from a large number of numerical simulations of the BLE, and the crosssection eq. (5.1), is evaluated by numerical integration. Figure 2 shows the total $\mathrm{K}^{+}$ production cross-section in ${ }^{12} \mathrm{C}+{ }^{12} \mathrm{C}$ collisions as a function of bombarding energy. The solid line and the dashed line in Figure 2 represents the result of calculations in the BL and the BUU approaches, respectively. These calculations are performed with a soft mean-field and by taking only the nucleon-nucleon channels into account. The BL model with fluctuations, in particular at low energies, gives much larger cross-sections than those obtained in the BUU description. The dots in Figure 2 represent the 


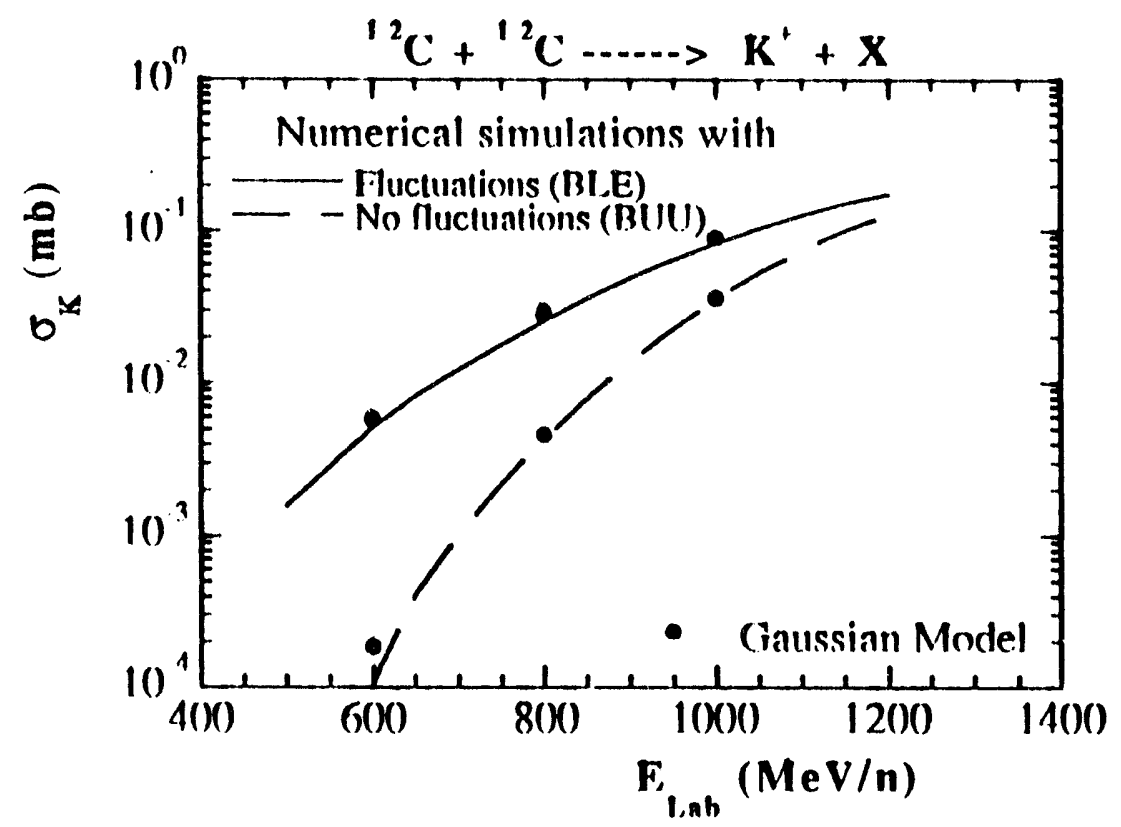

Figure 2. The $\mathrm{K}^{+}$production cross-section in ${ }^{12} \mathrm{C}+{ }^{12} \mathrm{C}$ collisions as a function of the bombarding energy. Shown are the BLE simulations (solid line), the BUU simulations (dashed line) and the Gaussian model calculations (dots). The calculations are performed with a soft mean-field. Taken from ref. [19].

calculations performed within the Gaussian model, which agree well with the numerical simulations.

\section{CONCLUSIONS}

The development of transport descriptions may lead to novel theoretical tools for understanding the complex reaction mechanism in heavy-ion collisions at intermediate energies. In particular, the recently developed BL model is a leading candidate theory for addressing such catastrophic phenomena as phase transitions and nuclear multifragmentations. Because of numerical complexity, however, 80 far a limited number of applications have been carried out. The lattice simulation of the BLE, proposed in ref. [12], is so computer intensive that the realistic applications to heavy-ion collisions are difficult to make. The projection method developed in ref. [13] is very efficient for realistic applications, however, one has to truncate the multipole expansion in momentum space rather arbitrarily. Therefore, there is a need for developing approximate methods for realistic applications of the BL model to heavy-ion collisions. One recent effort in this direction may provide a very powerful method for simulating the BLE in realistic problems [22].

Heavy-ion collisions at bombarding energies of a few $\mathrm{GeV} / \mathrm{n}$ give rise to highly compressed and excited nuclear matter during the initial stages of the collision. At these energies, the baryonic excitations and the mesonic degrees of freedom become important. In the framework of the Walecka-type field theory, the BL approach has been extended to the relativistic collisions [23]. It will be interesting to investigate the 
influence of correlations on the particle production mechanism and the collision dynamics at high energies using the relativistic BL model.

I wish to acknowledge my collaborators C. Gregoire, E. Suraud, D. Boilley, M. Belkacem and P. G. Reinhard, with whom I have had the pleasure of collaborating through various periods over the past several years. This work is supported in part by US-DOE grant DE-FG05-89ER40530.

\section{RGFGRENCES}

1. G. F. Bertsch and S. Das Gupta, Phys. Rep. 160 (1988) 190.

2. W. Cassing and U. Mosel, Prog. Part. Nucl. Phys. 25 (1990) 235.

3. S. Ayik and C. Gregoire, Phys. Lett. B212 (1988) 269; and Nucl. Phys. A513 (1990) 187.

4. J. Randrup and B. Remaud, Nucl. Phys. A514 (1990) 339.

5. P. G. Reinhard, E. Suraud and S. Ayik, Ann. Phys. 213 (1992) 204; and P. G. Reinhard and E. Suraud, preprint GANIL-P9107 (1991), Ann. Phys. (N.Y.) 216 (1992) 987.

6. S. Nakajima, Prog. Theor. Phys. 20 (1958) 948.

7. R. W. Zwanzig, Quantum Statistical Mechanics, ed. P. H. E. Meijer (Gordon and Breach, New York, 1966).

8. H. Risken, The Fokker-Planck Equation (Springer, Berlin, 1984).

9. M. Bixon and R. Zwanzig, Phys. Rev. 187 (1969) 267.

10. M. Colonna, Ph. Chomaz and J. Randrup, Nucl. Phys: A (1993) in press; J. Randrup, Interdisciplinary Workshop on Statistical Description of Transport in Plasma, Astro- and Nuclear Physics, Les Houchers, France (1992), Nova Science.

11. S. Ayik, E. Suraud, J. Stryjewski and M. Belkacem, Z. Phys. A337 (1990) 413; S. Ayik and D. Boilley, Phys. Lett. B276 (1992) 263 and 286 (1992) 482F; S. Ayik, submitted to Phys. Lett. (1993).

12. Ph. Chomaz, G. F. Burgio and J. Randrup, Phys. Lett. B254 (1991) 340; G. F. Burgio, Ph. Chomaz and J. Randrup, Nucl. Phys A529 (1991) 157; and F. Chapelle, et al., Nucl. Phys. A540 (1992) 227.

13. E. Suraud, S. Ayik, J. Stry jews and M. Belkacem, Nucl. Phys. A542 (1992) 141; S. Ayik, E. Suraud, M. Belkacem and D. Boilley, Nucl. Phys. A545 (1992) 35c; E. Suraud, S. Ayik, M. Belkacem and F.-S. Zhang, preprint GANIL-P9316 and submitted to Nucl. Phys. A(1993).

14. C. Gregoire, B. Remaud, F. Sebille, L. Vinet and Y. Raffray, Nucl. Phys. A465 (1987) 317.

15. F.-S. Zhang and E. Suraud, preprint YTTP/K-1G09 and submitted to Phys. Lett. B (1993).

16. D. R. Bowman, et al., Phys. Rev. Lett. 67 (1991) 1527.

17. C. A Ogilvie, et al., Phys. Rev. Lett. 67 (1991) 1214.

18. P. Danielewicz, Ann. Phys. 197 (1990) 154.

19. M. Belkacem, E. Suraud and S. Ayik, Phys. Rev. C47 (1993) R16.

20. W. Cassing, V. Mettag, U. Mosel and K. Niita, Phys. Rep. 188 (1990) 363.

21. J. Randrup and C. M. Ko, Nucl. Phys. A343 (1980) 519; and 411 (1983) 537.

22. J. Randrup and S. Ayik, preprint LBL-34484, and submitted to Nucl. Phys. A (1993).

23. S. Ayik, Phys. Lett. B265 (1991) 47. 

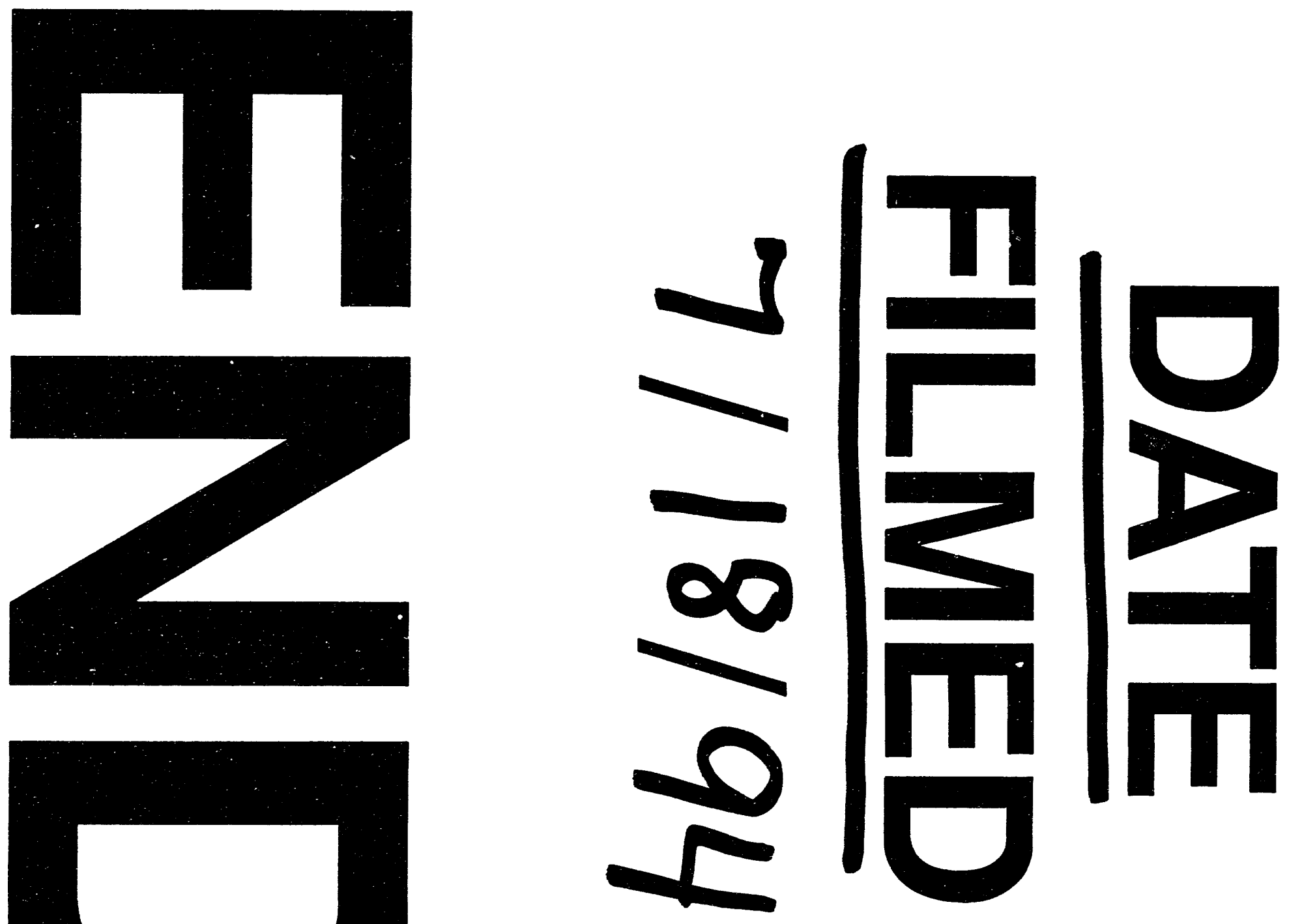
